\title{
Cost-effectiveness analysis of $5 \%$ lidocaine-medicated plaster compared with pregabalin for the treatment of post-herpetic neuralgia in China
}

\author{
Fei Zeng", Meng Wang", Daying Zhang \\ Department of Pain Medicine, The First Affiliated Hospital of Nanchang University, Nanchang, China \\ Contributions: (I) Conception and design: D Zhang; (II) Administrative support: F Zeng; (III) Provision of study materials or patients: F Zeng; (IV) \\ Collection and assembly of data: M Wang; (V) Data analysis and interpretation: F Zeng, M Wang; (VI) Manuscript writing: All authors; (VII) Final \\ approval of manuscript: All authors. \\ \#These authors contributed equally to this work. \\ Correspondence to: Daying Zhang. Department of Pain Medicine, The First Affiliated Hospital of Nanchang University, Nanchang, China. \\ Email: zdysino@163.com.
}

\begin{abstract}
Background Post-herpetic neuralgia (PHN) is the most common complication of herpes zoster and is defined as pain that lasts for one month or more after the outbreak itself heals. While the annual incidence of herpes zoster is approximately 3-5\%, 9-34\% of these patients will develop PHN. Approximately 30-50\% of these cases last for more than a year but some cases can persist for 10 years or more. To date, the economic burden of PHN in China has not been studied. The first-line topical therapy for PHN is application of lidocaine-medicated plasters (LMPs) which have shown good efficacy and tolerability. Furthermore, LMPs were added to China's National Health Insurance List in 2019, thereby significantly relieving the financial burden on patients. A cost-effectiveness analysis was performed on LMPs compared with pregabalin in the treatment of PHN to provide a reference for the basis for clinical treatments and health decisions in patients with PHN.
\end{abstract}

Methods: A Markov model was built according to the PHN disease characteristics. The efficacy data were extracted from a randomized controlled trial conducted in China, and the transition probability, utility value, and medical cost of each state in the model were collected through a systematic review of the literature and public databases. The outcome measure was cost per quality-adjusted life year (QALY) gained. The incremental cost-effectiveness ratios (ICERs) were calculated. Sensitivity analysis was conducted to confirm the robustness of the model.

Results: In the base case analysis, treatment for a 6-month period with pregabalin and lidocaine plasters led to a mean QALY gain of 0.34012 and 0.42543 , respectively, and mean incremental costs of 5,720 Chinese

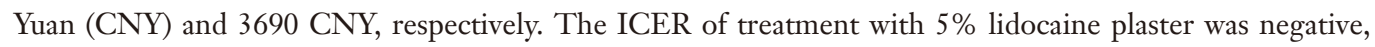
indicating that lidocaine plasters had absolute advantage. Monte Carlo simulation resulted in an estimate of $90 \%$ probability that the $5 \%$ lidocaine plaster treatment was cost-effective.

Conclusions: Within the Chinese medical and health system, LMPs can reduce the economic burden of patients with PHN. LMPs are more cost-effective and more efficient in absolute terms compared to the firstline treatment systemic drug pregabalin in the treatment of PHN.

Keywords: Post-herpetic neuralgia; pharmacoeconomic evaluation; cost-effectiveness analysis; Markov model; lidocaine-medicated plaster (LMP)

Submitted Feb 03, 2021. Accepted for publication Apr 04, 2021.

doi: $10.21037 / \mathrm{apm}-21-529$

View this article at: http://dx.doi.org/10.21037/apm-21-529 


\section{Introduction}

Herpes zoster is caused by the reactivation of the latent varicella zoster virus (VZV) and often occurs in people with low immunity, especially the elderly. The clinical manifestation of the $\mathrm{VZV}$ is varicella or occult infection after the initial infection, and it remains latent in the spinal dorsal root ganglion, craniocerebral sensory ganglion, or the intestinal meridian. Post-herpetic neuralgia (PHN) is the most common complication of herpes zoster and is defined as pain that lasts 1 month or more after the herpes zoster infection heals (1). The annual incidence of herpes zoster is approximately 3-5\% (2). Approximately 9-34\% of patients with herpes zoster develop PHN (3), and 30-50\% of those cases last for more than a year, with some cases persisting for 10 years or more (2).

At present, there is no cure for PHN, and management consists of pain relief and improving the quality of life. Common drugs for systemic treatment include gabapentin, pregabalin (PG), fentanyl, oxycodone, and tramadol. Topical drugs include lidocaine-medicated plasters (LMPs) and capsaicin (4).

LMPs block voltage-gated sodium channels and reduce the ectopic impulses of primary afferent nerves after injury, thus reducing pain in patients with PHN. Although LMPs satisfy the domestic need for a first-line topical medication for PHN and offer patients a new choice of treatment, they also bring a certain degree of economic burden to patients. The costs of managing PHN patients are significantly higher than those of herpes zoster patients without neuralgia. A study conducted in Latin America on patients aged 50 years and older with herpes zoster showed that the direct cost of PHN is 1,227 USD, the indirect cost is 773 USD, and the total cost is 2,001 USD (5). A study in Italy showed that the cost per case of PHN is 5,400 EUR (6). However, to date, the economic burden of PHN in China has not been examined.

It is important to match limited medical and health resources with true medical demand, as well as to ensure that the drugs listed on the Chinese government reimbursement list are both clinically efficient and costeffective. Pharmacoeconomic evaluations not only provide a basis for the selection of optimal and individualized treatments but can also effectively control the ballooning of medical and health expenses. In this study, a Markov model was established to conduct a cost-effectiveness analysis of LMPs compared with PG in the treatment of PHN.

We present the following article in accordance with the
CHEERS reporting checklist (available at http://dx.doi. org/10.21037/apm-21-529).

\section{Methods}

Using the existing evidence-based data, a Markov model was established for cost-effectiveness analysis from a perspective of China. The simulated target population of this study were post-herpetic neuralgia patients receiving different doses of LMPs and PG. The expected life years or quality-adjusted life years (QALYs) and medical expenses were estimated, and the incremental cost-effectiveness ratio (ICER) was calculated. Finally, sensitivity analysis was carried out. When the ICER of LMPs against PG was less than three times the per capita gross domestic product (GDP) of China in 2018 [198,018 Chinese yuan (CNY)] the treatment was deemed cost-effective.

\section{Markov state}

Markov model is widely used in disease models with multistate transition. In this study, the Markov state transition model was established based on the treatment plan of PHN disease according to the methods of Liedgens et al. (7) (Figure 1). The time horizon of the model was 6 months, the cycle was 1 month, and the model had 5 states. The patients transferred between the disease states according to their treatment responses every 30 days, and the states were as follows:

(I) Run-in with lidocaine or PG;

(II) Maintenance with lidocaine or PG;

(III) Run-in with lidocaine plus additional medication or PG plus additional medication;

(IV) Maintenance with lidocaine plus additional medication, or PG plus additional medication;

(V) Drop-out.

The run-in phase is the test dose period, which is the initial state. If there are no serious adverse reactions and the pain is well relieved, the patient will enter the maintenance state. If the pain is not effectively relieved, the patient will enter the "plus additional medication" state. If there are adverse reactions or treatment stops, the patient will enter the drop-out state. According to the 2016 Consensus of Chinese Experts in the Diagnosis and Treatment of PHN (1), the combination of drugs and minimally invasive interventional therapy can effectively relieve the pain of PHN. A retrospective analysis of the levels of evidence for minimally invasive interventional 


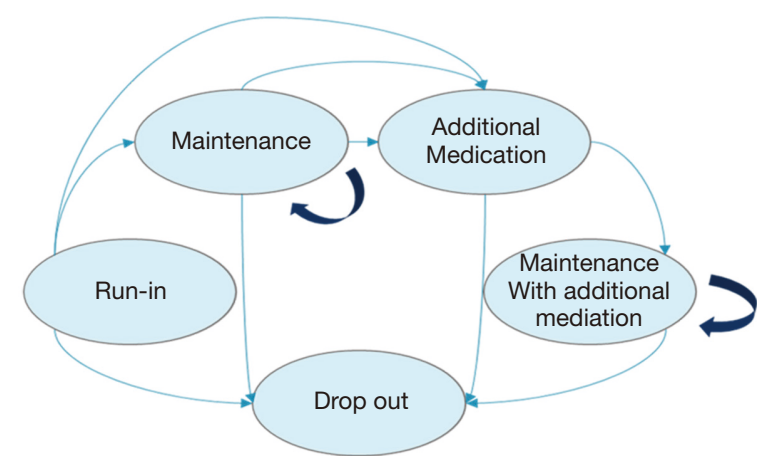

Figure 1 The structure of the Markov model.

therapy showed that common and reliable methods for PHN included nerve block, pulsed radiofrequency therapy, and nerve electrical stimulation. If the treatment strategy is effective, the patient will enter the maintenance state, and if it is not effective, the patient will enter the dropout state. The drop-out state is the state in which the use of these drugs and interventions are ineffective and other drugs such as gabapentin, duloxetine, tramadol, morphine, or capsaicin will be administered instead.

\section{Transition probability}

The data on transition probabilities between states in each treatment group were derived from a 2013 European study (7), from which the state transition probability data of a head-to-head study in the UK (8) were selected (Table 1). Due to the limited clinical data on domestic LMPs, there was insufficient information to obtain all the transition probabilities in this model. Therefore, the transition probabilities with LMP use mainly refer to foreign clinical trials. A comparison of the efficacy of domestic Phase III clinical trials (9) with that of foreign clinical trials (Table 2) found that, despite differences in the method of evaluating treatment efficacy, the efficacy of domestic LMPs was similar to that of foreign LMPs in terms of the evaluation cycle, end point, and effective rate. Therefore, it was deemed acceptable to refer to foreign efficacy data on LMP effectiveness in this model. In addition, in the scenario analysis (SA), a comparison was made in the input model of the available clinical data in China.

Patients assess pain using the numeric rating scale (NRS) and the visual analog scale (VAS). The NRS is a discrete scale from 0 to 10 , where 0 represents no pain and 10 indicates unbearable pain. The VAS uses a straight $100 \mathrm{~mm}$ horizontal line, where one end represents the absence of pain and the other end represents the worst possible pain. Patients quantify the intensity of their pain by drawing a vertical line at the place closest to their pain level.

\section{Treatment costs}

"Expenditure" in this study refers to the cost of drugs used for disease treatment and the cost of interventional therapy without pain relief. It does not include indirect costs and parts of direct costs, such as registration fees and hospitalization fees.

The price of the LMP covered by the National Medical Insurance Program is $19 \mathrm{CNY}$ per unit, with a maximum dose of 3 units per day. PG is administered at a maximum of $600 \mathrm{mg} /$ day, and is available as a pack of eight $75 \mathrm{mg}$ tablets at $82.9 \mathrm{CNY} /$ packet, or eight $150 \mathrm{mg}$ tablets at $141.18 \mathrm{CNY} /$ packet. The average doses of LMP and PG vary, with 1.71 units LMP and $488 \mathrm{mg}$ PG used in some clinical studies (10). Reports from Europe (7), the Netherlands, Germany, Scotland, and the United States suggested daily administration of 1.03 units of LMP. In China, the average clinical doses of both agents are lower, and therefore 1.0 unit LMP and $300 \mathrm{mg}$ PG were used in this study for comparative analysis.

The cost of each state is shown in Table 1. Additional medication is defined as the increased use of drug regimens such as duloxetine and tramadol, as well as nerve block due to inadequate pain relief. The total cost of this state is approximately $370 \mathrm{CNY}$ per month, according to expert interviews. The drop-out condition is when a user of a firstline drug withdraws from the program or switches to other drug regimens or nerve block surgery due to side effects or other reasons. The cost of this state is approximately $320 \mathrm{CNY} /$ month. It is recommended that PG should be gradually reduced for at least one week if it is to be stopped. Therefore, the cost of PG drop-out is $269 \mathrm{CNY}$ more than that of LMP (the cost of a low dose of $300 \mathrm{mg} / \mathrm{day}$ for 7 days is $269 \mathrm{CNY}$ ).

In pharmacoeconomic research, the process of converting the costs and health utilities at different times into the costs and health utilities at the same "time point" at a certain interest rate is called discount. Discounting was not included in this study due to the relatively short treatment time horizon of 6 months.

\section{Health utilities}

QALY is an important parameter to evaluate the quality of 
Table 1 Model input of Markov state

\begin{tabular}{|c|c|c|c|c|c|c|}
\hline Parameters & State & \multicolumn{2}{|c|}{ LMP } & \multicolumn{2}{|c|}{ PG } & - Source \\
\hline \multirow{4}{*}{$\begin{array}{l}\text { Cost per } \\
\text { cycle (CNY) }\end{array}$} & Run-in & 570 & 975 & 1,151 & 1,873 & \multirow{10}{*}{$\begin{array}{l}\text { LMP SA: Chinese Journal } \\
\text { of Clinical Research [Aiping } \\
\text { Wang et al. (9)]. PG: clinical } \\
\text { trial data [Baron et al. (10)]. } \\
\text { LMP BCA: clinical trial data } \\
\text { [Baron et al. (10), Hans } \\
\text { et al. (11)] }\end{array}$} \\
\hline & Maintenance & 570 & 975 & 1,151 & 1,873 & \\
\hline & Run-in plus additional medication & 940 & 1,345 & 1,521 & 2,243 & \\
\hline & Drop out & \multicolumn{2}{|c|}{320} & \multicolumn{2}{|c|}{589} & \\
\hline \multirow[t]{2}{*}{ Utility } & Run-in & BCA: 0.916 & SA: 0.899 & \multicolumn{2}{|c|}{0.791} & \\
\hline & Maintenance & BCA: 0.916 & SA: 0.899 & \multicolumn{2}{|c|}{0.791} & \\
\hline \multirow[t]{4}{*}{$\begin{array}{l}\text { Transition } \\
\text { probability }\end{array}$} & $\begin{array}{l}\text { Dropout due to side effects during run-in } \\
\text { phase }\end{array}$ & BCA: 0.026 & SA: 0.0168 & 0.2 & 235 & \\
\hline & Remaining on treatment after run-in phase & \multicolumn{2}{|c|}{ BCA: 0.633} & \multicolumn{2}{|c|}{0.468} & \\
\hline & Discontinuation during maintenance phase & \multicolumn{2}{|c|}{ BCA: 0.044} & \multicolumn{2}{|c|}{0.123} & \\
\hline & $\begin{array}{l}\text { Adding in additional medication during } \\
\text { maintenance }\end{array}$ & \multicolumn{2}{|c|}{ BCA: 0.068} & \multicolumn{2}{|c|}{0.062} & \\
\hline
\end{tabular}

BCA, base case analysis; SA, scenario analysis; LMP, lidocaine medicated plaster; PG, pregabalin.

Table 2 Efficacy of lidocaine-medicated plaster in domestic phase III clinical trials and foreign clinical trials

\begin{tabular}{lcc}
\hline Item & Foreign clinical trial (10) & Domestic phase trial (9) \\
\hline Control & LMP vs. PG & LMP vs. placebo \\
Observation period & 4 weeks & 4 weeks \\
Scoring method & NRS & VAS \\
End point and effective rate & LMP: drop by $30 \%: 57.8 \%$; drop by $50 \%: 35.6 \%$ & LMP: drop by $30 \%: 56.07 \% ;$ drop by $50 \%: 36.45 \%$ \\
Adverse reactions & $18.7 \%$ & $22.5 \%$ \\
\hline
\end{tabular}

LMP, lidocaine medicated plaster; PG, pregabalin; NRS, numeric rating scale; VAS, visual analogue scale.

life. The quality-of-life adjustment weight used for QALYs is calculated as the health utility value. The health utility value is the weight of a certain health state relative to complete health, an index to evaluate the satisfaction of a certain health status, and a comprehensive index reflecting individual health status. The range of values is 0 to 1 , where 0 represents death and 1 represents full health. The health utility values in this study were derived from the literature referenced by the European Summary Research Institute mentioned above. The utility value of complete relief state is 1 , and that of persistent pain is 0.55 (8). Therefore, the utility value of each state of the treatment regimen was calculated as the weighted average value according to the adverse reaction rate (Table 1).

\section{Sensitivity analysis}

Following the recommendations in the China Guidelines for Pharmacoeconomic Evaluations, the uncertainty caused by data collection and study assumptions were addressed 
Table 3 The results of the cost-effectiveness analysis

\begin{tabular}{|c|c|c|c|c|c|c|}
\hline Strategies & Eff & IncrEff & Cost (CNY) & IncrCost & ICER & Avg CE \\
\hline 300 mg PG & 0.34012 & & $5,719.8$ & & & $16,817.3$ \\
\hline 1.0 unit LMP & 0.42543 & 0.08531 & $3,690.3$ & $-2,029.5$ & Dominant & $8,674.3$ \\
\hline \multicolumn{7}{|c|}{1.71 unit LMP vs. 488 mg PG } \\
\hline 1.71 unit LMP & 0.42543 & 0.08531 & $5,686.3$ & $-2,372.5$ & Dominant & 13,366 \\
\hline
\end{tabular}

Eff, effectiveness; IncrEff, incremental effectiveness; IncrCost, incremental cost; ICER, incremental cost-effectiveness ratio; Avg CE, average cost-effectiveness ratio; LMP, lidocaine medicated plaster; PG, pregabalin.

by sensitivity analysis to consider the impact of different parameter changes on the running results of the model. This method ensures the stability and reliability of the research results.

In the one-way sensitivity analysis, the low values ( $75 \%$ as the lower limit) and the high values $(125 \%$ as the upper limit) of different data were calculated to examine the impact of the range on the results. The upper limit of the health utility value does not exceed 1.0. The variation ranges of specific variables in the one-way sensitivity analysis are shown in Table S1.

Probability sensitivity analysis refers to sampling different parameters according to a preset distribution to find their effect on the outputs of the model in the sampling range. The Monte Carlo simulation is used to analyze the probability sensitivity and record the outputs of the model. Conducting 1,000 iterations ensures that the uncertainties associated with the input of the model are taken into account. The distribution of each variable in the probability sensitivity analysis is shown in Table S1, and the standard deviation is $10 \%$.

\section{Statistical analysis}

TreeAge Pro 2011 (TreeAge Software, Inc., Williamstown, MA) and Microsoft Excel ${ }^{\circledR} 2019$ software were used for statistical analysis. The upper and lower limits of model inputs are used in one-way sensitivity analysis. The utilities were assumed to be sampled from Beta distribution, and costs were sampled from gamma distribution in probabilistic sensitivity analysis. The one-way sensitivity analysis and probabilistic sensitivity analysis results are respectively presented as Tornado diagram and incremental cost effectiveness ratio scatter plot.

\section{Results}

\section{Cost-effectiveness analysis}

During the 6-month treatment period, $300 \mathrm{mg}$ PG and 1.0 unit LMP (19 CNY/unit) provided 0.34012 and 0.42543 QALYs, respectively (Table 3). The cumulative drug cost and cumulative additional treatment costs were 5,719.8 $\mathrm{CNY}$ and 3,690.3 CNY, respectively. The average costeffectiveness ratio of $300 \mathrm{mg}$ PG was 16,817.3 CNY/ QALY, while that of 1.0 unit LMP was 8,674.3 CNY/ QALYs. Therefore, the cost-effectiveness ratio of the LMP was significantly lower than that of $300 \mathrm{mg}$ PG. When comparing 1.71 units LMP with $488 \mathrm{mg}$ PG, the ICER showed that 1.71 units LMP was a dominant strategy, suggesting that LMPs have an absolute advantage over PG.

\section{One-way sensitivity analysis}

The sensitivity analysis was carried out by taking $75 \%$ of the variables in the model as the lower limit and $125 \%$ as the upper limit. The tornado diagram shows that the utility of maintenance with LMPs, utility of run-in with LMPs, and the utility of maintenance with LMPs plus additional medication had the greatest influence on the model outputs, but there was no influence on the research conclusion (Figure 2).

\section{1,000 Monte Carlo simulations}

In this study, Monte Carlo simulations were carried out with 1,000 iterations to find the differences in outputs based on different transition probabilities. Probability sensitivity analysis can simulate and analyze multiple uncertain factors simultaneously according to the probability distribution of 
Tornado Diagram (Net Benefit, 1.0 unit LMPs vs. 300 mg PG)

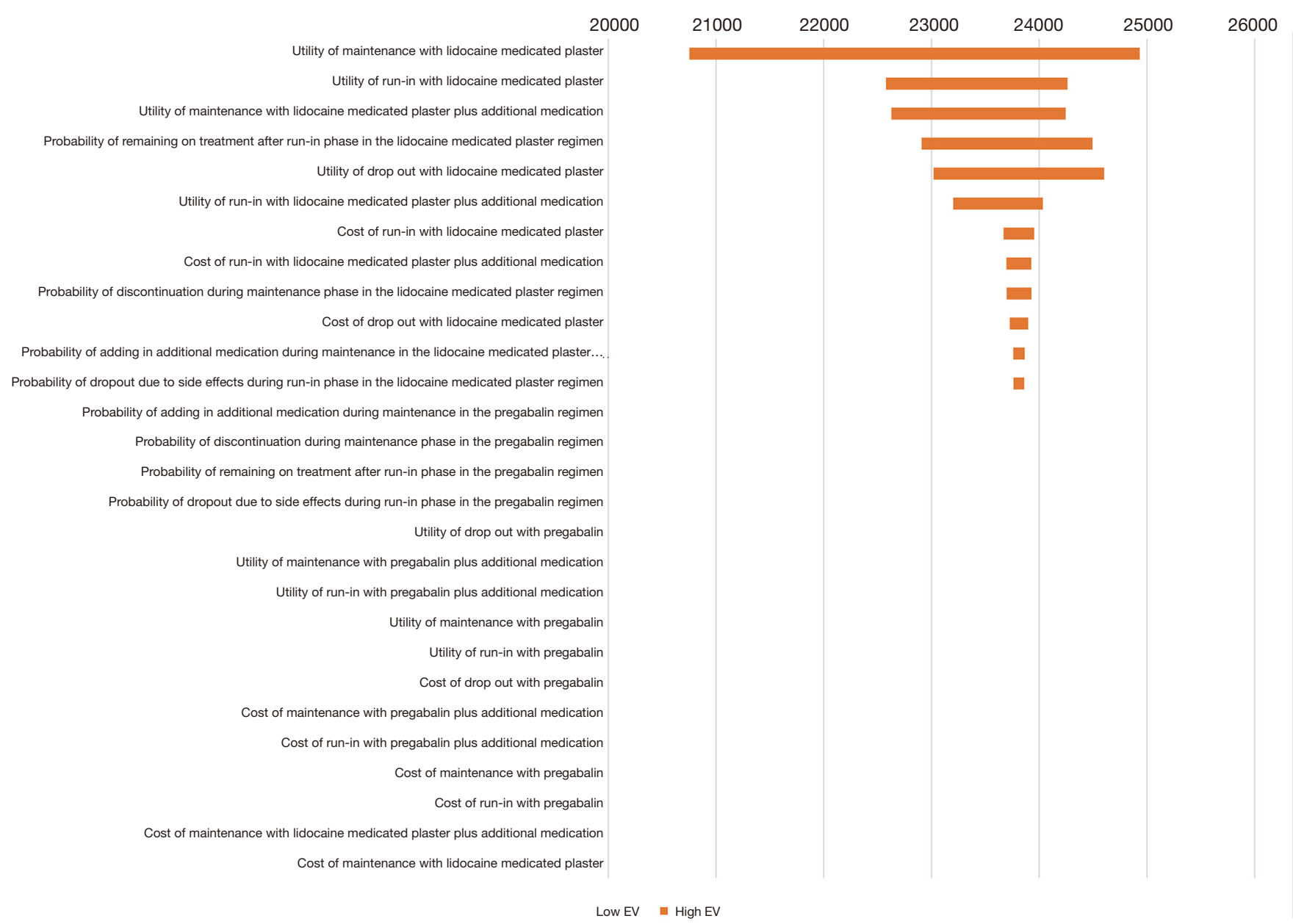

Figure 2 Tornado diagram comparing 1.0 unit lidocaine-medicated plaster (LMP) with $300 \mathrm{mg}$ pregabalin (PG). EV, expected value.

variables (Figure 3). Most of the points were scattered in the fourth quadrant of the plot, indicating that in most cases, LMPs were more effective and cost less compared to PG. This study had stable analysis outputs, and lidocaine had an absolute advantage over PG.

\section{Scenario analysis}

\section{Scenario analysis 1}

The control group was analyzed with reference to the scenario analysis in the European Health Economics study (7).

In the scenario analysis of the European Health Economics study (7), the United Kingdom, Spain, Austria, and Portugal all cited 1.1 unit of LMP in comparison to $488 \mathrm{mg} \mathrm{PG}$, as 1.1 unit of LMP was considered to be a frequently used dose in the clinical setting. According to the input model, the total cost of 1.1 unit of LMP during the 6-month treatment period was 5,686.3 $\mathrm{CNY}$, and the average cost-effectiveness ratio was 13,366.0 CNY/QALY. The total cost of $488 \mathrm{mg}$ PG was $8,058.8 \mathrm{CNY}$, and the average cost-effectiveness ratio was 23,694.3 CNY/QALY. The ICER of 1.1 unit of LMP was negative compared to that of $488 \mathrm{mg}$ PG. The results of this scenario analysis are shown in Table 4.

\section{Scenario analysis 2}

Data on the cost-effectiveness of LMPs in China.

The early detachment rate in a Phase III clinical trial of LMPs in China was $1.68 \%$, and the proportion of adverse reactions was $22.5 \%$. The cost-effectiveness model (Table 4) showed that 1.0 unit of LMP resulted in 0.41968 QALYs 


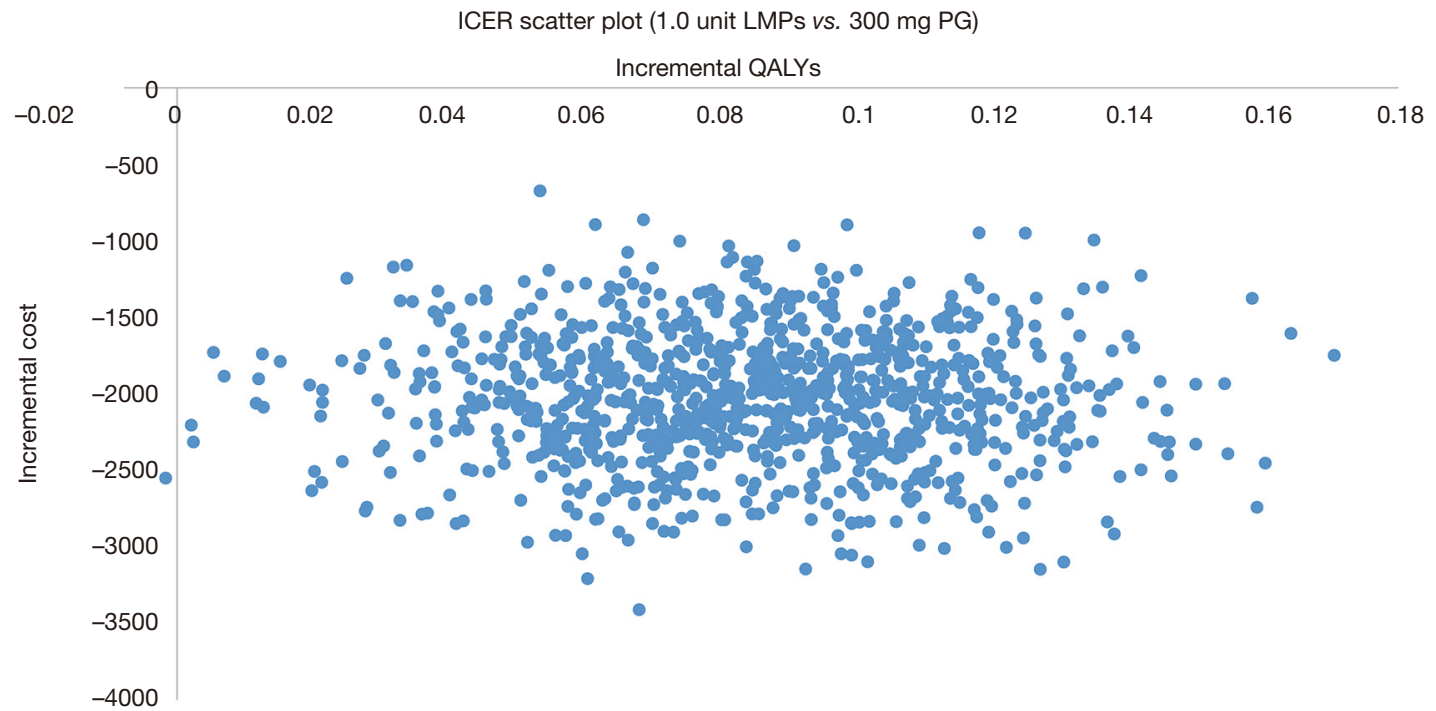

Figure 3 Incremental cost effectiveness ratio (ICER) scatter plot comparing 1.0 unit lidocaine-medicated plaster (LMP) with $300 \mathrm{mg}$ pregabalin (PG). The unit of incremental cost is Chinese yuan (CNY) in the y-axis. QALY, quality-adjusted life year.

Table 4 Scenario analysis

\begin{tabular}{|c|c|c|c|c|c|c|}
\hline Scenario & Eff & IncrEff & Cost (CNY) & IncrCost & IC/IE & Avg CE \\
\hline $488 \mathrm{mg}$ PG & 0.34012 & & $8,058.80$ & & & $23,694.30$ \\
\hline 1.1 unit LMP & 0.42543 & 0.08531 & $5,686.30$ & $-2,372.50$ & $-27,810.31$ & $13,366.00$ \\
\hline \multicolumn{7}{|c|}{ Scenario analysis 2: 1.0 unit LMP vs. 300 mg PG } \\
\hline 1.0 unit LMP & 0.41968 & 0.07957 & $3,710.30$ & $-2,009.50$ & $-25,255.67$ & $8,840.70$ \\
\hline
\end{tabular}

Eff, effectiveness; IncrEff, incremental effectiveness; IncrCost, incremental cost; IC/IE, incremental cost-effectiveness ratio; Avg CE, average cost-effectiveness ratio; LMP, lidocaine medicated plaster; PG, pregabalin.

during the 6-month treatment period, the cumulative drug cost and additional treatment cost totaled 3,710.3 CNY, and the average cost-effectiveness ratio was $8,840.7 \mathrm{CNY} /$ QALY, which was lower than that of the $300 \mathrm{mg}$ PG group.

\section{Discussion}

PHN, known as "immortal cancer", is an intractable nerve pain condition that afflicts middle-aged and elderly individuals. Under the long-term influence of severe pain, $80 \%$ to $90 \%$ of patients will have emotional and psychological abnormalities such as anxiety, depression, and even suicidal tendencies. PHN has always been a very thorny problem in the clinic. At present, common treatments include oral medicine, topical ointments, and nerve block agents. Topical LMPs have been used abroad for more than 20 years. This strategy is the first-line treatment for PHN and is recommended in the treatment guidelines of many countries. This drug was unavailable in China until 2018, when LMPs sold under the product name DeBaiNing $^{\circledR}$ was listed on the market. This new treatment option will open new possibilities for the treatment of PHN patients in China.

Clinical trials have shown that LMPs deliver enhanced efficacy, better safety, and improved patient satisfaction and quality of life compared to PG (10). Despite the advantages of lidocaine, the financial burden to the patient must be considered. Cost-effectiveness analysis (CEA) investigates 
both cost and efficacy, and therefore provides a reference for optimizing clinical drug regimens.

In this investigation, a pharmacoeconomic evaluation was conducted comparing LMPs with PG. The results showed that 1.0 unit of LMP had superior efficacy and reduced costs and therefore, it had an absolute advantage compared with $300 \mathrm{mg}$ PG. This is similar to the scenario analysis comparing 1.1 unit of LMP and $488 \mathrm{mg}$ PG. In the past, LMPs were relatively expensive, which caused a certain financial burden on patients. When the 2019 edition of the National Healthcare Catalog included LMPs, this effectively reduced the financial burden on patients, resulting in improved clinical effectiveness and quality of life. The results of this study demonstrated that LMPs are a very costeffective treatment option for $\mathrm{PHN}$ patients in China.

One-way sensitivity analysis showed that the utility of maintenance with LMPs, utility of run-in with LMPs, and the utility of maintenance with LMPs plus additional medication had the greatest influence on the model outputs, but the outputs of the one-way sensitivity analysis did not change the conclusion that LMPs are more cost-effective than PG. In addition, 1,000 iterations of Monte Carlo simulations based on the probability distribution of model variables showed that most of the points focused on the fourth quadrant. This suggested that LMPs have a lower cost and better efficacy than PG.

Our study model is based on ten earlier European studies, but the outcomes differed markedly. The difference between our research and the earlier European research is that the research perspective is different. China has just been able to use lidocaine-medicated plasters in 2018, and its economy has not been studied. As far as we know, this study is the first time to conduct a cost-effectiveness analysis of the first-line therapeutic drugs lidocaine-medicated plasters and pregabalin in the treatment of post-herpetic neuralgia from the perspective of China. Our study showed that $300 \mathrm{mg}$ PG and 1.0 unit of LMP provided 0.34012 and 0.42543 QALYs, respectively, during the 6-month treatment period. The cumulative drug cost and cumulative additional treatment costs were 5,719.8 CNY (€730.1) and 3,690.3 CNY (€471), respectively, which is lower than that reported in the European analysis (the average costs of PG and LMPs were $€ 516.5$ and $€ 1,075.4$, respectively). Additionally, the cumulative QALY gains of LMPs and PG were higher in this study than in the European analysis (the average cumulative QALY gains of PG and LMPs were 0.2787 and 0.3265 , respectively) (12-15). This suggested that the efficacy of LMPs has improved and that their inclusion in China's National Health Care Policy has made the price more acceptable. This greatly reduces the financial burden on patients with PHN. Therefore, LMPs are much more cost-effective than PG for PHN treatment in China.

As with any analysis model, this study has certain limitations. First, the transfer probability selected in the model is based on foreign research data, although the efficacy of domestic LMPs is similar to that of foreign LMPs in terms of evaluation period, end point, and effectiveness. However, the differences between domestic and foreign scenarios may affect the final analysis results, causing the results to be merely similar rather than completely consistent. In addition, the health utility values were derived from the data of foreign clinical trials, which may also lead to differences between the results calculated from foreign and domestic data. However, it did not affect the conclusion from the qualitative analysis which is that LMPs are the dominant strategy.

\section{Conclusions}

With the inclusion of LMPs on China's National Medical Insurance List, the cost of medicines for patients with PHN has been greatly reduced, thereby lightening the economic burden of patients. Our CEA showed that LMPs have lower cost, improved clinical effectiveness, significantly better cost-effectiveness, and higher economic value compared to PG, and are therefore worthy of clinical use.

\section{Acknowledgments}

Funding: None.

\section{Footnote}

Reporting Checklist: The authors have completed the CHEERS reporting checklist. Available at http://dx.doi. org/10.21037/apm-21-529

Data Sharing Statement: Available at http://dx.doi. org/10.21037/apm-21-529

Conflicts of Interest: All authors have completed the ICMJE uniform disclosure form (available at http://dx.doi. org/10.21037/apm-21-529). The authors have no conflicts of interest to declare. 
Ethical Statement: The authors are accountable for all aspects of the work in ensuring that questions related to the accuracy or integrity of any part of the work are appropriately investigated and resolved. This study was based on a literature review and modeling techniques. This study did not require approval by an institutional research ethics board.

Open Access Statement: This is an Open Access article distributed in accordance with the Creative Commons Attribution-NonCommercial-NoDerivs 4.0 International License (CC BY-NC-ND 4.0), which permits the noncommercial replication and distribution of the article with the strict proviso that no changes or edits are made and the original work is properly cited (including links to both the formal publication through the relevant DOI and the license). See: https://creativecommons.org/licenses/by-nc-nd/4.0/.

\section{References}

1. Yu S, Wan Y, Wan Q, et al. Consensus of Chinese experts on diagnosis and treatment of post-herpetic neuralgia. Chinese Journal of pain Medicine 2016;22:161-7.

2. Kawai K, Gebremeskel BG, Acosta CJ. Systematic review of incidence and complications of herpes zoster: towards a global perspective. BMJ Open 2014;4:e004833.

3. Kost RG, Straus SE. Postherpetic neuralgia--pathogenesis, treatment, and prevention. N Engl J Med 1996;335:32-42.

4. Attal N, Cruccu G, Baron R, et al. EFNS guidelines on the pharmacological treatment of neuropathic pain: 2010 revision. Eur J Neurol 2010;17:1113-e88.

5. Rampakakis E, Pollock C, Vujacich C, et al. Economic Burden of Herpes Zoster ("culebrilla") in Latin America. Int J Infect Dis 2017;58:22-6.

6. Gialloreti LE, Merito M, Pezzotti P, et al. Epidemiology and economic burden of herpes zoster and post-herpetic neuralgia in Italy: a retrospective, population-based study. BMC Infect Dis 2010;10:230.

7. Liedgens H, Obradovic M, Nuijten M. Health

Cite this article as: Zeng F, Wang M, Zhang D. Costeffectiveness analysis of $5 \%$ lidocaine-medicated plaster compared with pregabalin for the treatment of post-herpetic neuralgia in China. Ann Palliat Med 2021;10(4):4493-4501. doi: 10.21037/apm-21-529 economic evidence of $5 \%$ lidocaine medicated plaster in post-herpetic neuralgia. Clinicoecon Outcomes Res 2013;5:597-609.

8. Ritchie M, Liedgens H, Nuijten M. Cost effectiveness of a lidocaine $5 \%$ medicated plaster compared with pregabalin for the treatment of postherpetic neuralgia in the UK: a Markov model analysis. Clin Drug Investig 2010;30:71-87.

9. Wang A, Wang X. Efficacy and safety of lidocaine cataplasms $5 \%$ for the treatment of post-herpetic neuralgia. Journal of Practical Dermatology 2015;8:181-4.

10. Baron R, Mayoral V, Leijon G, et al. $5 \%$ lidocaine medicated plaster versus pregabalin in post-herpetic neuralgia and diabetic polyneuropathy: an open-label, non-inferiority two-stage RCT study. Curr Med Res Opin 2009;25:1663-76.

11. Hans G, Sabatowski R, Binder A, et al. Efficacy and tolerability of a $5 \%$ lidocaine medicated plaster for the topical treatment of post-herpetic neuralgia: results of a long-term study. Curr Med Res Opin 2009;25:1295-305.

12. Nuijten MJ, Pais BR, Liedgens H, et al. PND7 Costeffectiveness of a lidocaine plaster relative to pregabalin in the treatment of post-herpetic neuralgia in The Netherlands. Value in Health 2008;11:A601.

13. Liedgens H, Hertel N, Gabriel A, et al. Cost-effectiveness analysis of a lidocaine $5 \%$ medicated plaster compared with gabapentin and pregabalin for treating postherpetic neuralgia: a german perspective. Clin Drug Investig 2008;28:583-601.

14. Dakin HA, Nuijten MJC, Liedgens H, et al. NE4 costutility analysis evaluating lidocaine $5 \%$ medicated plaster relative to gabapentin for postherpetic neuralgia in Scotland. Value in Health 2007;10:A9-A10.

15. Dakin H, Nuijten $M$, Liedgens $H$, et al. Cost-effectiveness of a lidocaine $5 \%$ medicated plaster relative to gabapentin for postherpetic neuralgia in the United Kingdom. Clin Ther 2007;29:1491-507.

(English Language Editor: J. Teoh) 


\section{Supplementary}

Table S1 Variation range and distribution in the sensitivity analysis

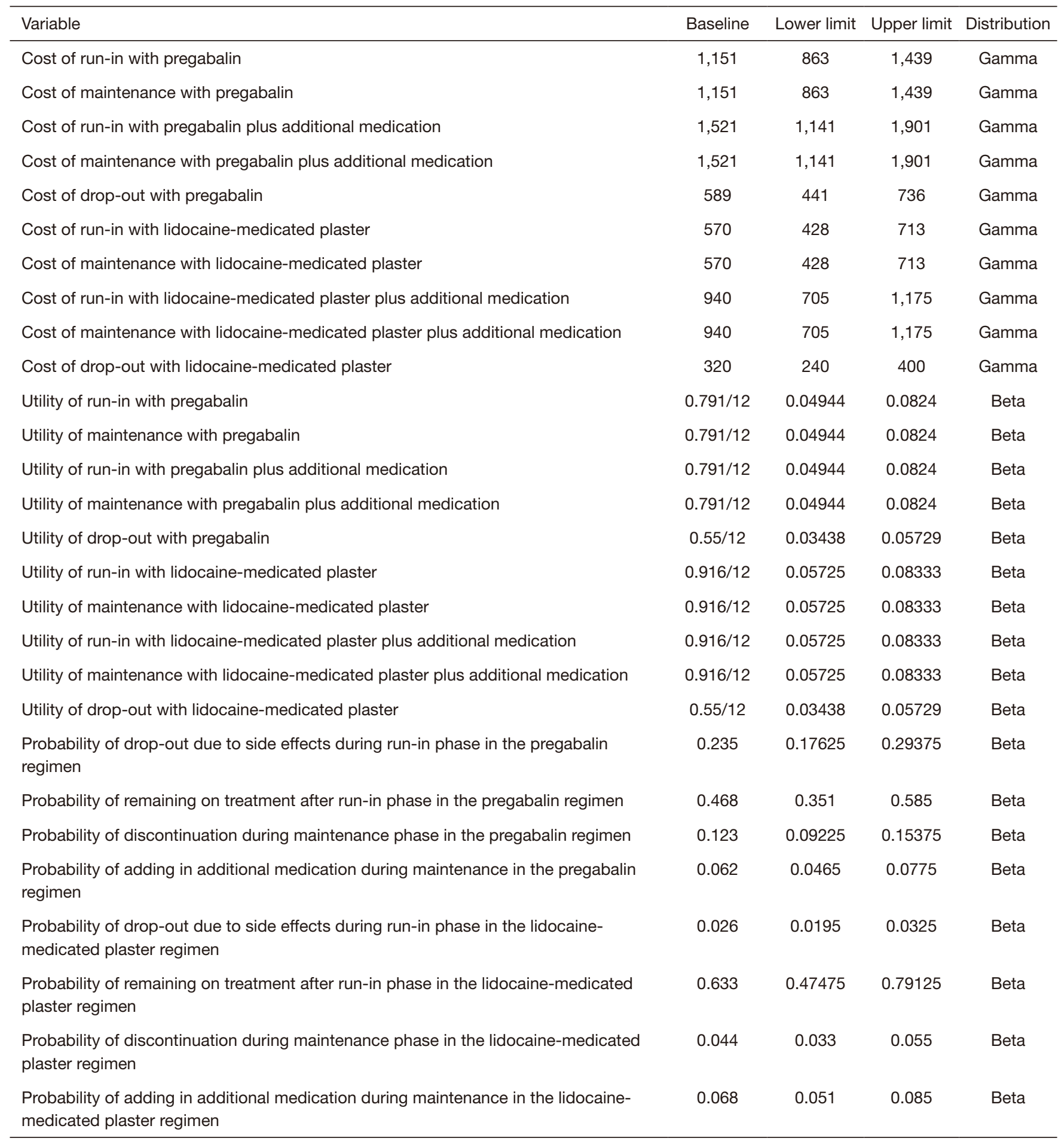

